\title{
Stimulus-based versus performance-based measurement of auditory backward recognition masking
}

\author{
DAVID C. FOYLE and CHARLES S. WATSON \\ Boys Town National Institute, Omaha, Nebraska
}

\begin{abstract}
Estimates of the time required for the extraction of pitch information from auditory memory have been made using the auditory backward recognition masking (ABRM) technique. With this technique, the ability to recognize the pitch of the first of two sequential tones is determined for various delays between the tones. Most investigators have used fixed stimulus values and have measured performance in terms of $P(C)$, the proportion of correct responses. Use of this measure, proportion correct, as the dependent variable is shown in the present studies to yield, for ABRM, duration estimates that are different from those obtained when the same data are evaluated in stimulus terms for a fixed level of performance. The time course of information extraction from auditory memory was found to extend over approximately $100 \mathrm{msec}$ when measured in stimulus terms, in contrast to the $250 \mathrm{msec}$ that has been reported from data expressed as a proportion of correct responses to a fixed stimulus value. The apparently disparate results previously reported are shown to be mutually consistent if monotonic psychophysical functions are assumed to provide an adequate description of performance in ABRM tasks. Several reasons are proposed for preferring the stimulus-based measures of ABRM.
\end{abstract}

The recent resurgence of interest in the mechanisms by which sensory information is processed has included consideration of a variety of aspects of memory for sounds. The classical concept of the duration of auditory memory is the length of time the details of a sound remain available, as reflected in a listener's ability to judge that a new sound was the same as or differed in some way from one heard previously. Another, much shorter term aspect of auditory memory is the length of time a sound must be available before some specific attribute can be appreciated or identified. This aspect of memory has been studied in experiments in which auditory processing is assumed to be subject to termination by the presentation of a succeeding stimulus.

The time-course of information extraction from auditory memory has been investigated by Massaro (1970, 1976) and various other authors (Leshowitz \& Cudahy, 1973; Loeb \& Holding, 1975; Sparks, 1976; Yost, Berg, \& Thomas, 1976), using a task that Massaro termed auditory backward recognition masking (ABRM). In a typical single-interval version of the ABRM task (Figure 1),

The research conducted and the preparation of this article were supported by NIH/NINCDS Grant NS 20606 at Indiana University and NIH/NINCDS Grant NS 14637 at Boys Town National Institute. Portions of this research were presented at the 105th Meeting of the Acoustical Society of America, May 1983, Cincinnati. The authors wish to thank Dominic Massaro, Donald Robinson, and two anonymous reviewers for their helpful comments on earlier versions of this paper. The authors also thank Theresa Langer and Leslie King for their assistance with the figures. Reprint requests should be sent to the authors, who are now at the Department of Speech and Hearing Sciences, Indiana University, Bloomington, IN 47405. two tones are separated by some variable time delay, $\Delta \mathrm{t}$. The listener's task is to identify the pitch of the first tone as higher or lower than the second. The assumption is that the second tone, called the masker, interferes with, or masks, the first tone by terminating its processing. By varying $\Delta \mathrm{t}$, the time course of information extraction from auditory memory is estimated in terms of the functional relationship between performance and $\Delta t$. If $\Delta t$ is long, the listener is assumed to have the necessary time required to extract the pitch information from auditory memory,

$$
\begin{aligned}
& \text { Single-Interval } \\
& \text { Identification }
\end{aligned}
$$

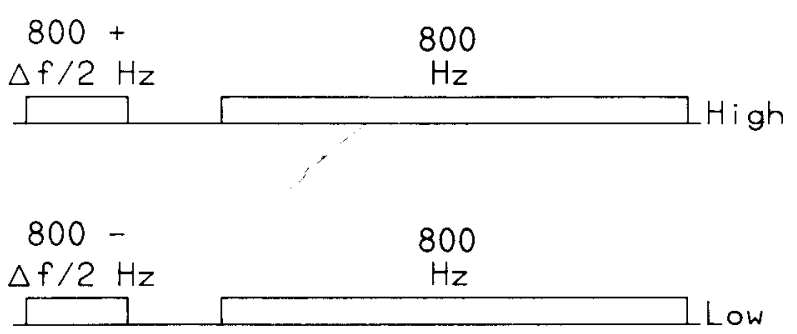

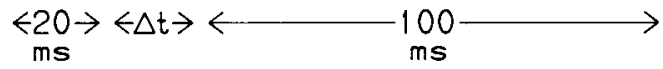

Figure 1. Schematic display of the two possible two-tone sequences. The variable delay between the 20 -msec first tone and the 100 -msec masker tone was $\Delta t$ msec (with specific values of $\Delta t$ of $0,20,40$, 80, 160, and 320 msec in Experiment 1). The frequency difference between the two possible first tones is $\Delta \mathrm{f} \mathrm{Hz}$. In the first experiment, the first tone was either 780 or $820 \mathrm{~Hz}(\Delta \mathrm{f}$ of $40 \mathrm{~Hz}$ ). 
as shown by the ability to decide whether the first tone was higher or lower in pitch than the second. Degraded performance as $\Delta t$ becomes shorter is assumed to reflect insufficient time to extract the pitch information from the first tone before the masker disrupts its processing.

Massaro and others (for a review, see Massaro, 1976) have obtained data that support these assumptions: Listeners perform well when $\Delta t$ is on the order of 150-300 msec, whereas performance may be severely degraded for values of $\Delta \mathrm{t}$ between 0 and $40 \mathrm{msec}$. It is difficult, however, to evaluate the duration of ABRM when it is expressed in terms of $\mathrm{P}(\mathrm{C})$, the proportion of correct responses, due to the nonlinear relation between that metric and stimulus dimensions. In the past, ABRM functions in the single-interval task have been expressed in terms of proportion correct as a function of $\Delta \mathrm{t}$. Typically, Massaro and his associates (e.g., Kallman \& Massaro, 1983) have estimated, by visual inspection, that the ABRM effect lasts about $250 \mathrm{msec}$. That is, performance in the pitch-identification task approaches asymptote for masking delays of about 250 msec. $^{\text {. }}$.

The results of ABRM experiments, and the interpretation of those results, have provoked considerable controversy almost since the first reports by Massaro and his co-workers (Massaro, 1970; Massaro \& Kahn, 1973). The degraded performance for even fairly large values of $\Delta t$ appeared to some authors to be at odds with psychoacoustic studies of other forms of backward masking and of pitch discrimination (Leshowitz \& Cudahy, 1973). Several experiments were reported showing that although the ABRM effects could be replicated, they were greatly reduced, if not eliminated, by prolonged training (Loeb \& Holding, 1975; Sparks, 1976). It was also proposed that the extended time course of learning in these experiments, and perhaps the actual form of the ABRM functions, might have been the result of the high levels of stimulus uncertainty that result from randomization of large sets of stimulus conditions within the same blocks of trials (Watson, Kelly, \& Wroton, 1976; Yost et al., 1976). In addition, Yost et al. reported that ABRM effects could be obtained under only one of several psychophysical methods with which they attempted to reproduce Massaro's results.

One characteristic of the published investigations of ABRM that distinguishes most of those studies from contemporary psychoacoustic research is the reliance on performance measures, generally the proportion of correct judgments, as the primary dependent variable. The more common procedure, particularly when the purpose of experiments is to determine the limits of auditory capabilities, is the determination of stimulus measures associated with some specified level of performance [i.e., "'thresholds,"' the stimulus levels required to achieve performance specified in terms of constant values of proportion correct, $d^{\prime}, P(C)_{\max }, P(A)$, or one of the other criterion-independent measures developed in the context of the theory of signal detectability (Green \& Swets,
1966/1974)]. The use of stimulus measures as dependent variables in most recent psychoacoustic research is probably based on the ease with which results expressed in those terms can be related to other aspects of auditory processing (signal-to-noise ratios, integration times, critical bandwidths, etc.) within various theoretical frameworks.

Another reason for avoiding performance measures as dependent variables may be the likelihood, when those measures are used, of misjudging the relative difficulties of various conditions in parametric studies. The more primitive functional relation underlying both stimulusbased or performance-based dependent variables is the psychometric function from which either type of measure can be derived. One goal of ABRM research is to determine a listener's ability to correctly identify the pitch of brief tone pulses with various delays between those pulses and a following masker. That goal is entirely consistent with either performance- or stimulus-based experimental methods and treatments of the resulting data. In the former case, the ABRM effects would be expressed as changes in proportion correct for various delays between test tone and masker $(\Delta t)$. In the latter, the same effects could be described in terms of the difference $(\Delta f$, in hertz), between two discriminable test-tone frequencies, for each value of $\Delta t$. It is obvious that a parametric study in which psychometric functions relating proportion correct judgments to $\Delta f$, for various values of $\Delta t$, could be replotted to yield either representation of the data.

It might seem unlikely that such a simple variation as shifting from a performance-based to a stimulus-based dependent variable could make a great difference in the general results of ABRM experiments, but this alternative approach seemed worthwhile for two reasons. First, ABRM has been suggested as a factor in auditory and temporal processing, and its properties expressed in stimulus terms could be more easily related to the acoustical features of speech or other temporally varying sounds of theoretical interest. Second, the relation between stimulusand performance-based measures would be extremely simple, with one measure predictable from the other by the most trivial of calculations, only if the underlying psychometric functions relating performance measures to $\Delta f$ were linear and of a common slope. This, however, is clearly not the case for families of sigmoid-shaped functions, and therefore, without actually generating the appropriate data, it is not possible to predict the form of isoperformance functions from the widely published isostimulus ABRM functions.

The two goals of the present studies were: First, to determine the size of the ABRM effect in stimulus terms, and from those measures to estimate quantitatively the time constant for the extraction of information from auditory memory; and second, to make these measurements using psychophysically well-trained listeners in a task in which relatively few stimulus conditions were intermixed, that is, under relatively low stimulus uncertainty. 


\section{EXPERIMENT 1}

\section{Method}

Subjects. Three college-age listeners, screened for normal hearing, were paid to serve as subjects. Each daily session lasted $1 \mathrm{~h}$. One listener had previously been tested in an unrelated forwardmasking experiment; the other two subjects were naive listeners. One additional listener, also with extensive forward-masking experience, did not finish the experiment because he was unable to perform above-chance levels even for the longest $\Delta t$ values. ${ }^{2}$ The subjects participated in a total of 1,300 trials during three 1 -h sessions, with all 1,300 trials reported here.

Stimuli and Procedure. The typical single-interval identification task was used, as shown in Figure 1. On each trial, a 20-msec tone (either 780 or $820 \mathrm{~Hz}$, yielding a $\Delta$ f of $40 \mathrm{~Hz}$ ) was followed by a $100-\mathrm{msec}, 800-\mathrm{Hz}$ tonal masker. A variable delay, $\Delta \mathrm{t}$ (randomly chosen from the set: $0,20,40,80,160$, or $320 \mathrm{msec}$ ), separated the two tones. The listener's task was to identify, by depressing a button, whether the first tone was lower $(780 \mathrm{~Hz})$ or higher $(820 \mathrm{~Hz})$ than the $800-\mathrm{Hz}$ masker.

Each trial began with a 250 -msec warning light, followed by a 250 -msec delay. The two tones, separated by $\Delta \mathrm{t} \mathrm{msec}$, were presented and immediately followed by a 1,500 -msec response interval. After the response interval, a 500-msec feedback light was illuminated above the correct button. The intertrial interval was 500 msec.

A PDP-11/23 computer controlled all timing, randomization, and stimulus presentation. Tones were generated with independent Tektronix FG 503 voltage-controlled oscillators under computer control. The subjects listened in a partitioned sound-attenuated booth, with the tones presented in the right ear only of Telephonics TDH-39 headphones. All tones were presented at $80 \mathrm{~dB}$ SPL and gated with rise-decay times of $2.5 \mathrm{msec}$.

After each 100 trials, the subjects were informed of their performance. In each 1-h session, the subjects typically completed $600-700$ trials.

\section{Results and Discussion}

Figure 2 shows the individual listeners' results averaged across the first 450 (approximately) trials on the ABRM task (collected during the first hour of testing). The results are similar to those typically found after 2 to $5 \mathrm{~h}$ of testing in the ABRM task (e.g., Kallman \& Mas-

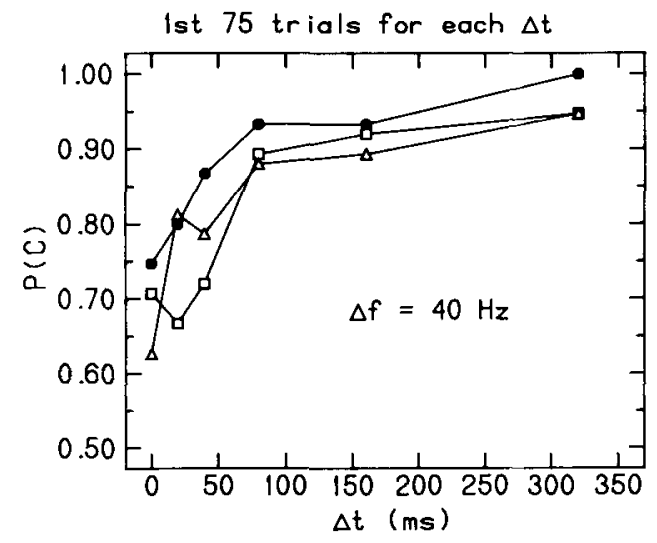

Figure 2. Data from Experiment 1 using a 20-msec tone, 100-msec masker, and $\Delta f$ of $40 \mathrm{~Hz}$. Each listener's data $[P(C)]$ is represented by one of the functions. Each data point is based on the first 75 trials at each $\Delta t$ value.

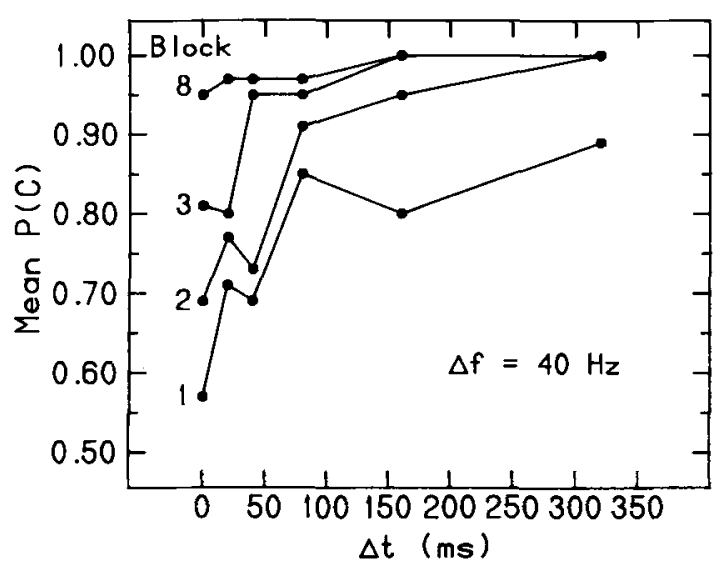

Figure 3. Mean $P(C)$ for the three listeners in Experiment 1 as a function of $\Delta t$. Data were partitioned into chronological blocks of 25 trials each. Data from the first three blocks and the final, or eighth, block only are shown. Each data point is based on 75 trials (three subjects, 25 trials each).

saro, 1983; Massaro, 1975; Massaro \& Kahn, 1973), but differ in two respects. First, all three listeners are performing above $P(C)=0.90$ at the longest $\Delta t$ of $320 \mathrm{msec}$, whereas previously reported subjects often have done less well at long values of $\Delta t$. Second, the ABRM functions for these listeners are somewhat steeper and appear to approach asymptote sooner.

As mentioned earlier, there have been several reports of significant training effects in the ABRM task (Loeb \& Holding, 1975; Sparks, 1976). Our data, likewise, showed a fairly dramatic improvement as testing progressed, as can be seen in Figure 3. This figure shows the average performance for each of the first three blocks of 25 trials per $\Delta t$ and for the 8th (final) block. After only 200 trials per $\Delta \mathrm{t}(2.5 \mathrm{~h}$ of testing), these subjects were performing perfectly at the longer values of $\Delta t$, and well above $P(C)=0.90$, even at $\Delta t$ of $0 \mathrm{msec}$. These subjects, however, were still exhibiting some ABRM (although ceiling effects obscure its actual magnitude), as reflected in less-than-perfect performance for the smaller values of $\Delta t$.

There is an apparent discrepancy between these results and those previously reported in the literature. The present data show comparable ABRM functions only in the first hour of testing, whereas other investigators have found ABRM effects after $4 \mathrm{~h}$ or more of testing. One possible explanation of the rapid learning in the present experiment is that the task may have been unusually easy in terms of the listeners' opportunities to develop optimal listening and decision strategies. Stimulus uncertainty, although not minimal, was low, with only 12 different stimuli (two test tones, and six $\Delta t$ values). The ABRM experiments in the literature have tended to have much larger levels of stimulus uncertainty (e.g., trial-to-trial selection from among 32 stimuli in Massaro, 1975; 48 in Massaro, Cohen, \& Idson, 1976; and 126 in Kallman \& Massaro, 1979). 
Because of the use of proportion correct as the dependent variable in this experiment, the results of testing could be assessed only for values of $\Delta t$ at which initial performance was poor. The second experiment was conducted to map the time course of the extraction of information from auditory memory in stimulus terms for specified levels of performance, thus avoiding the problems caused by the nonlinear relation between proportion correct and $\Delta t$ (range effects).

\section{EXPERIMENT 2}

\begin{abstract}
Method
Subjects. The same three listeners continued in this experiment after completing Experiment $1 .^{3}$

Stimuli and Procedure. The trial structure, tone durations, and values of $\Delta t$ were the same as those used in Experiment 1 , but $\Delta f$ was varied by an adaptive-tracking procedure (Levitt, 1971). The adaptive algorithm used was one for which the stimulus values presented would converge on $\mathrm{P}(\mathrm{C})=0.707$ performance. For each subject, six interleaved adaptive tracks (one for each $\Delta \mathrm{t}$ ) were maintained by the computer. The adaptive-tracking procedure determined, independently for each subject, the value of $\Delta f$ to be presented on each trial. Twenty possible values of $\Delta f$ could be presented, ranging in approximately equi-log steps from 2 to $60 \mathrm{~Hz}$. Trial structure was identical to that in Experiment 1, with the exception that the response interval was changed to $2,000 \mathrm{msec}$ (to preclude speed-accuracy limitations on performance for more difficult conditions). Data were collected in six daily 1 -h sessions.
\end{abstract}

\section{Results and Discussions}

Performance on this task became stable after the first few hundred trials, as evidenced by exponential fits to the data. All analyses were conducted on the final two-thirds of the data $(2,600$ total trials per subject $){ }^{4}$

A measure of discriminability, $d^{\prime}$, was calculated for each specific value of $\Delta f$ and $\Delta t$, individually for each listener. This yielded six psychometric functions (one per value of $\Delta t$ ) for each subject. These data were fit by linear psychometric functions in the space, $\log \Delta f \times \log \mathrm{d}^{\prime}$, using a least squares regression method (Egan, Lindner, \& McFadden, 1966), with each point weighted by the appropriate number of observations.

From the fitted psychometric functions, threshold estimates $\left(\Delta f\right.$ in hertz for $\left.d^{\prime}=1.0\right)$ were calculated, and are shown in Figure 4. As can be seen, performance is worst at the shortest values of $\Delta t$, but improves as $\Delta t$ is lengthened. The just-discriminable frequency difference is about $12.5 \mathrm{~Hz}$ at a $\Delta \mathrm{t}$ of $0 \mathrm{msec}$, improving to about $6.5 \mathrm{~Hz}$ at $320 \mathrm{msec}$. This frequency difference at $320 \mathrm{msec}$ is not quite as small, but is in the range of the thresholds reported by Moore (1973) for isolated tones (using two highly trained observers). The criterion measure, beta (see Green $\&$ Swets, 1966/1974), was calculated for each value of $\Delta t$, averaging over only those values of $\Delta \mathrm{f}$ near $\mathrm{d}^{\prime}=1.0$ $(0.70$ to 1.30$)$. These values of beta suggest that the subjects were using near-optimal decision strategies in the task. The Levitt (1971) tracking algorithm itself converged on similar threshold values of $\Delta \mathrm{f}$, as would be expected from the values of the criterion, beta, in Figure 4.
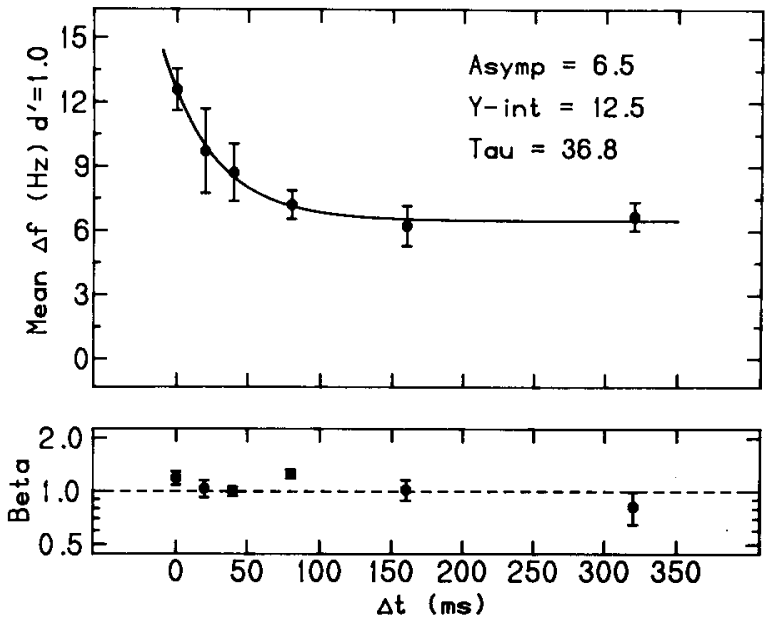

Figure 4. The upper panel shows $d^{\prime}=1.0$ average threshold estimates (see text) for the three subjects in Experiment 2 as a function of $\Delta t$. The three-parameter exponential fit and resulting parameters (asymptote, y-intercept, and tau) are shown. Error bars are the calculated standard error of the mean. The lower panel shows the criterion index, beta, for $\Delta f$ values near $d^{\prime}=1.0$ as a function of $\Delta t$. The dashed line at 1.0 represents the optimum criterion.

Although these listeners did exhibit backward masking, it appears to be a considerably briefer and smaller scale effect when plotted in stimulus terms than has been previously estimated: a degradation of about $6 \mathrm{~Hz}$, primarily occurring between values of $\Delta t$ of 0 and $100 \mathrm{msec}$. Leshowitz and Cudahy (1973) in a two-alternative forcedchoice version of the task found ABRM to be about a 9$\mathrm{Hz}$ effect. (For 20-msec test tones, those authors reported $75 \%$ level thresholds of about $17 \mathrm{~Hz}$ at $0 \mathrm{msec}$, decreasing to about $8 \mathrm{~Hz}$ at approximately $150 \mathrm{msec}$.) Thus, the ABRM effects, measured in proportion correct and expressed as changes from near-chance to near-perfect performance, may correspond to as little as a $6-\mathrm{Hz}$ change in threshold values, or about 0.0075 in terms of $\Delta f / f$.

The threshold estimates in Figure 4 were fit by a threeparameter exponential function (see Collected Algorithms from ACM, Inc., 1976, Algorithm 295). Tau, the time constant or rate of decay, was calculated to be $36.8 \mathrm{msec}$. Since $3 \times$ tau represents the time required for this function to decay $95 \%$ from the starting value toward asymptote, ABRM was essentially nonexistent beyond about $110 \mathrm{msec}$. This quantitatively estimated 110 -msec duration for the ABRM effect is less than half of the $250 \mathrm{msec}$ previously suggested (e.g., Kallman \& Massaro, 1983; Massaro, 1972a).

In summary, the finding of several earlier studies of an ABRM effect in the single-interval task was replicated. The effect, however, when measured in stimulus terms, appears to be much smaller (a degradation of threshold values of $\Delta f$ of about $6 \mathrm{~Hz}$ ) and of shorter duration $(110 \mathrm{msec})$ than has previously been reported.

The current estimates of ABRM have implications concerning the nature of auditory sensory memory and its effects on auditory processing. First, since the ABRM ef- 
fect is so small in stimulus terms, it is not likely to have significant effects on speech processing. (Kallman \& Massaro, 1983, have also recently made this point on the basis of other data.) Second, past conclusions based on the published isoperformance ABRM data are that a specific acoustical event represented in auditory sensory memory is fairly fragile and is "overwritten" by later arriving stimuli, and that this period of fragility lasts for $250 \mathrm{msec}$. The present estimates expressed in stimulus terms indicate that information in auditory sensory memory is not as fragile, as shown by low thresholds for $\Delta t$ of $0 \mathrm{msec}$, nor is the period during which it can be disrupted as long, as has been suggested.

\section{GENERAL DISCUSSION}

Some disagreement about backward-masking functions may have been provoked by features that are specific to the form of those functions when ABRM is expressed in changes in the proportion of correct responses. For example, Leshowitz and Cudahy (1973) have commented about the failure of many studies to obtain near-perfect performance at the longest $\Delta t$ values. In general, however, no investigator has discussed the general form of the underlying ABRM functions in $P(C)$ space. It might be expected that these functions should be traditional psychometric functions (S-shaped ogives derived from cumulative normal curves) with chance performance $[\mathrm{P}(\mathrm{C})$ $=0.50]$ at a value of zero of the independent variable, increasing to perfect performance $[\mathrm{P}(\mathrm{C})=1.00]$ at some large value of that variable. Clearly, this is not the predicted performance when the independent variable is the masker delay $(\Delta t)$. There is no a priori reason to expect chance performance at a $\Delta \mathrm{t}$ of $0 \mathrm{msec}$. A $\Delta \mathrm{t}$ of $0 \mathrm{msec}$, is merely a condition with no gap between the test tone and the masker, not a condition with no signal in terms of signal detection theories (e.g., Green \& Swets, $1966 / 1974$ ). Similarly, there is no reason to expect perfect performance, for any arbitrary value of $\Delta f$, as $\Delta t$ is made large.

What, then, is the nature of the underlying theoretical ABRM functions in $P(C) \times \Delta t$ space for a fixed value of $\Delta \mathrm{f}$ ? Column 1 of Figure 5 refers to fits of the data obtained in Experiment 2. The upper panel of Column 1 shows the average fitted psychometric functions to our data for the six values of $\Delta t$ [following translation of values of $d^{\prime}$ to $P(C)_{\max }$. The panel below it shows a family of iso- $\Delta f$ (isostimulus) ABRM functions (with $\Delta f$ as a parameter) that were derived by taking vertical "slices" at various values of $\Delta \mathrm{f}$ through the six psychometric functions in the upper panel. In this manner, the form of the ABRM function could have been estimated, had the experiment been conducted with various fixed values of $\Delta f$.

These derived ABRM functions appear exponential in form. At a $\Delta t$ of $0 \mathrm{msec}$, performance is at some level above chance, whereas for the longer values of $\Delta t$, performance can be less than perfect. Of particular interest, the ABRM function estimated for $\Delta \mathrm{f}$ of $12 \mathrm{~Hz}$ is similar in all respects to that recently reported by Kallman and Massaro (1983) for a subgroup of particularly acute listeners, with a $\Delta \mathrm{f}$ of about $8 \mathrm{~Hz}$.

Another important detail of the ABRM functions can also be seen in the middle panel of Column 1 in Figure 5. These iso- $\triangle \mathrm{f} A B R M$ functions, when examined in $P(C)$ $\times \Delta t$ space, do not approach asymptotes at the longest values of $\Delta t$ (except for the two functions derived from the smallest values of $\Delta f$, due to a crossing of the psychometric functions for 160 and $320 \mathrm{msec}$, and for the largest values of $\Delta \mathrm{f}$, due to ceiling effects). The ABRM functions continue to increase with longer values of $\Delta t$. This is consistent with the data shown in Figure 2, and has been found in other studies as well (e.g., Massaro, 1973). Therefore, attempting to estimate the value of $\Delta t$ at which functions like these "approach asymptote" does not appear to be a fruitful exercise.

In contrast to plotting performance for fixed stimulus values, functions can be plotted as in the bottom panel of Column 1 in Figure 5, showing iso-d' (isoperformance) ABRM functions. These are arrived at by taking horizontal "slices" through the fitted psychometric functions.

Based on the data of Experiment 2 (shown in Figure 4 and in the upper panel of Column 1 in Figure 5), two plausible models of theoretical psychometric functions are shown in the uppermost panels of Columns 2 (increasingslope model) and 3 (constant-slope model) of Figure 5. In both models, the six functions have the values for $\mathrm{d}^{\prime}=1.0$ obtained from the fitted exponential function shown in Figure 4 (ranging between 12.5 and $6.5 \mathrm{~Hz}$, for values of $\Delta t$ between 0 and $320 \mathrm{msec}$ ). The two models differ in the assumption concerning the relationship of slope to $\Delta t{ }^{5}$ The increasing-slope model assumes that the slopes increase as $\Delta t$ increases from 0 to $320 \mathrm{msec}$. Systematic changes in the slopes of psychometric functions have been obtained in many psychophysical studies and probably reflect decreased variance in the easier conditions, as represented by the leftmost psychometric functions, and increased variance, due to shifts in listening strategies, criterion shifts, etc., in the rightmost functions, representing the most difficult conditions. ("Easy" and "difficult" as used here are obviously meaningful only in stimulus terms.) The actual slopes shown in the increasing-slope model were estimated from the regression line relating slopes to $\Delta t$ from Experiment 2 . The constant-slope model assumes that the slopes are constant (the mean slope of the six psychometric functions obtained in Experiment 2 was used).

The increasing-slope model, with underlying psychometric functions of increasing slope for increasing $\Delta t$ and exponentially decreasing thresholds $\left(\mathrm{d}^{\prime}=1.0\right)$, gives rise to the iso- $\Delta \mathrm{f}$ (isostimulus) and iso-d' (isoperformance) ABRM functions shown in Column 2 of Figure 5. One interesting note regarding the increasing-slope model is that performance $\left[\mathrm{P}(\mathrm{C})_{\max }\right.$ in the iso- $\Delta \mathrm{f}$ plot in Column 2 of Figure 5] for many of the values of $\Delta \mathrm{f}$ is still increas- 


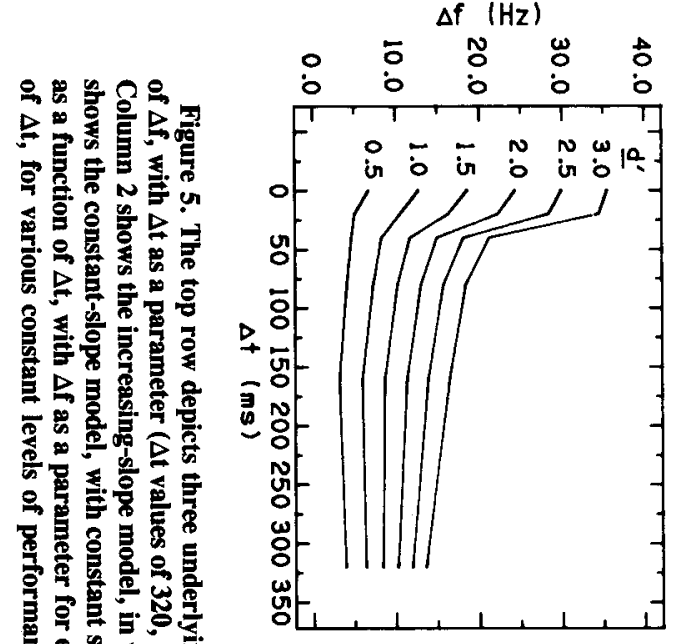

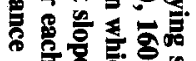

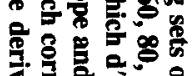

국을 욜

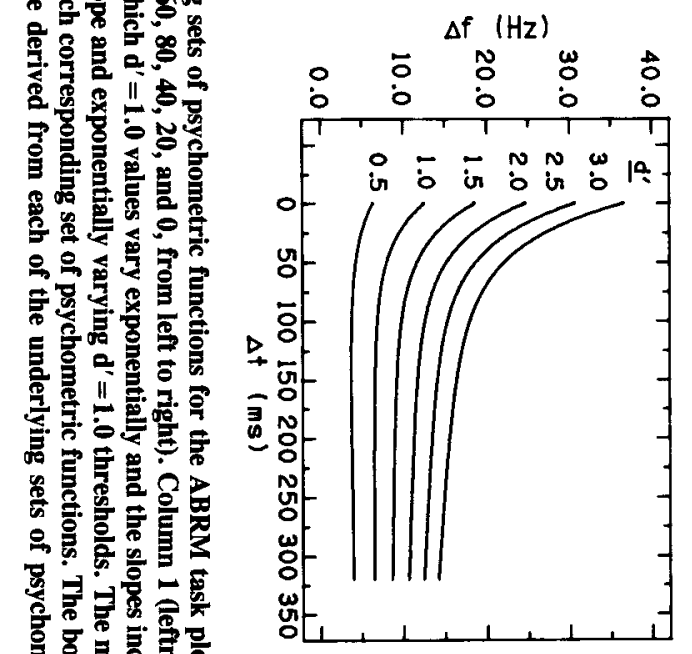

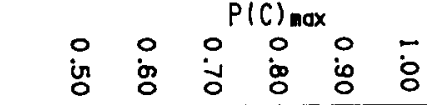
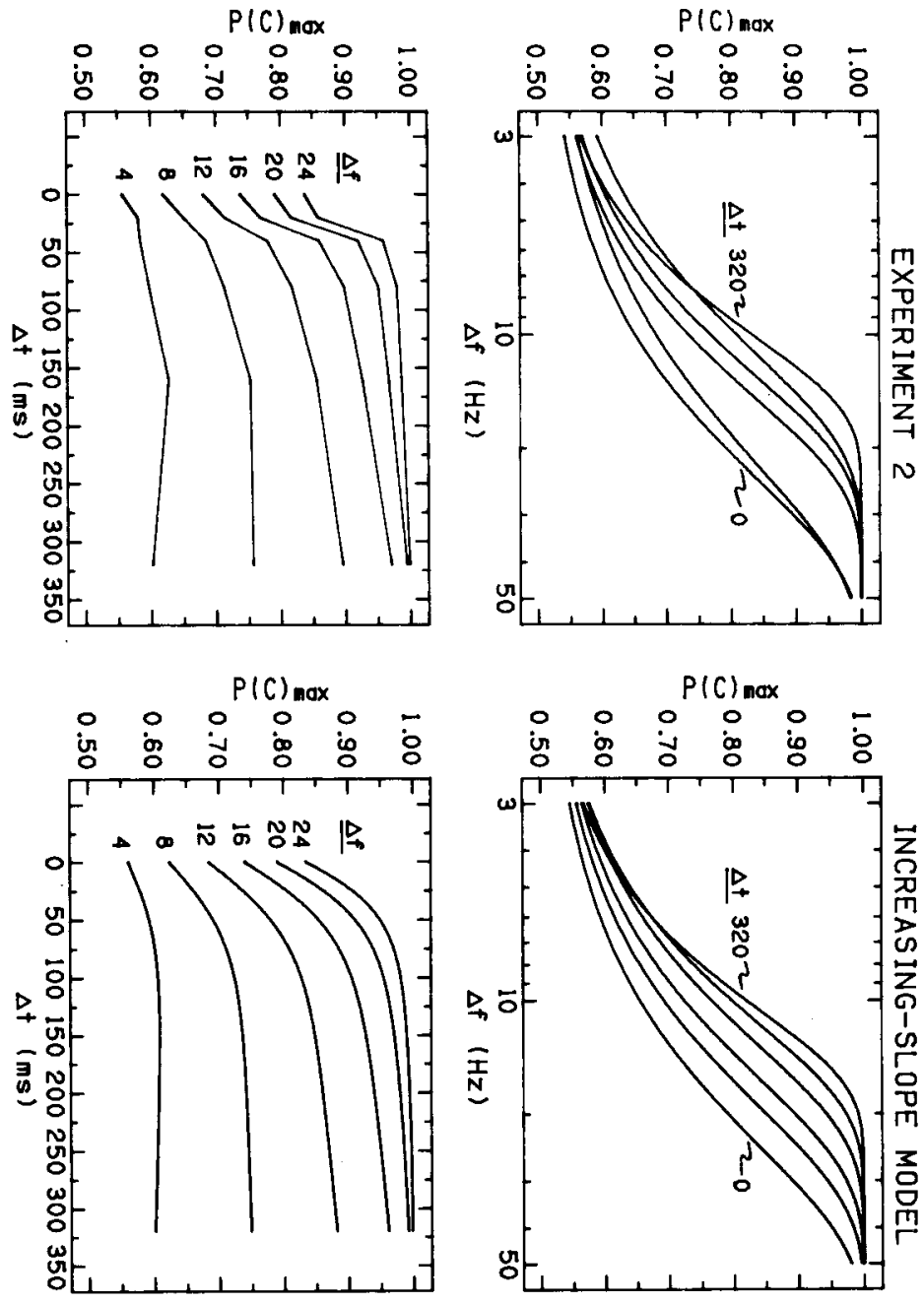

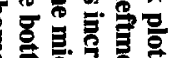

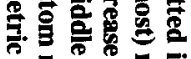

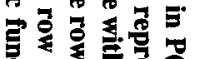
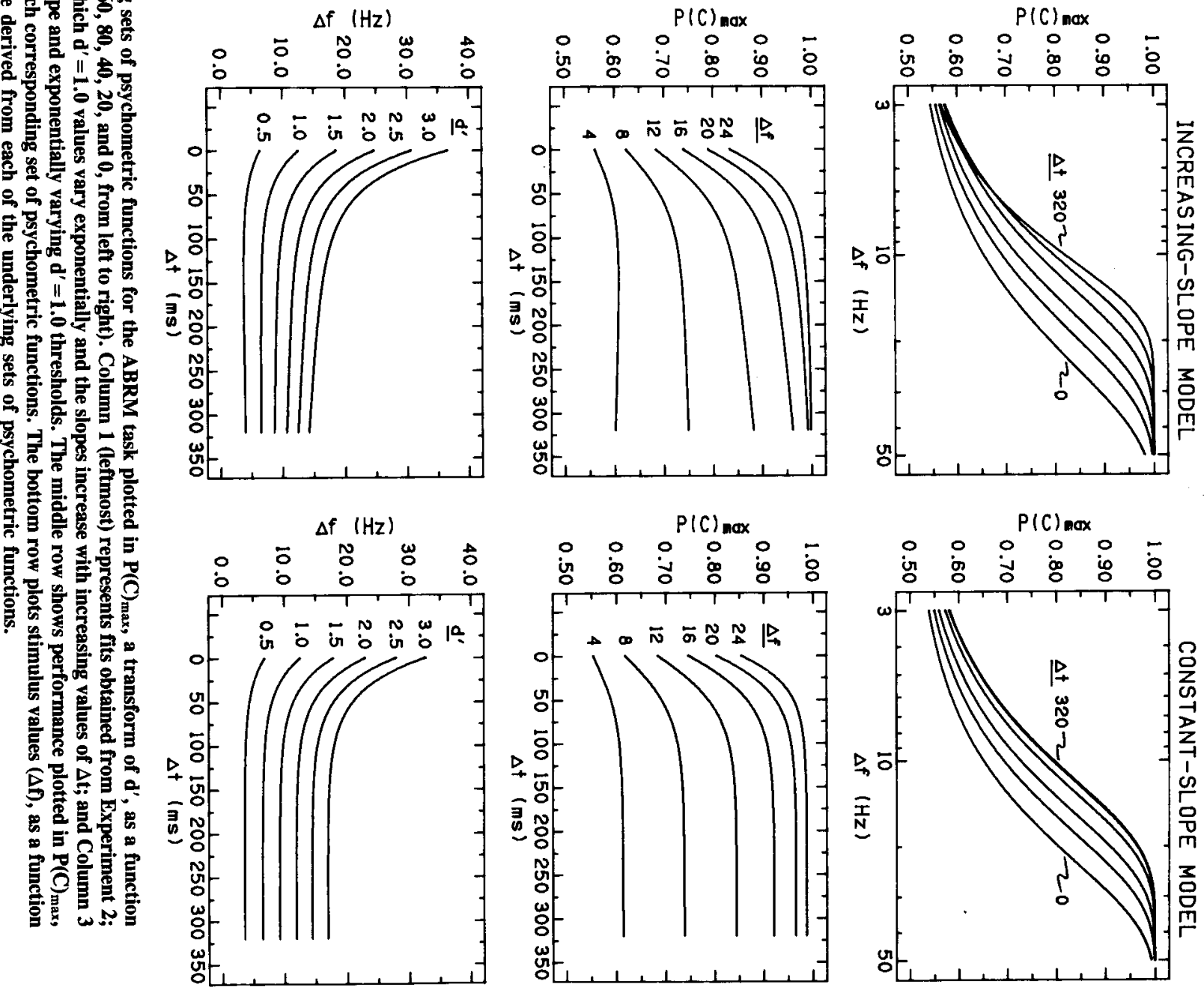

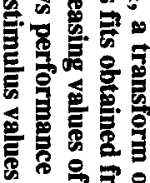

它을

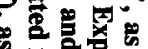

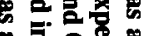

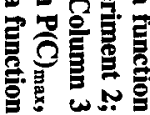

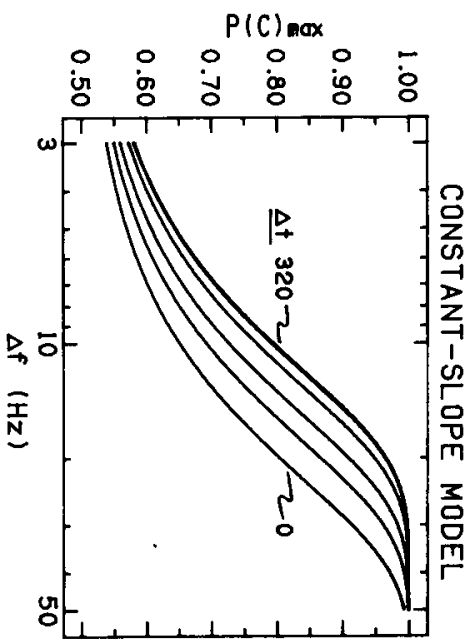


ing at the longer values of $\Delta \mathrm{t}(100$ to $320 \mathrm{msec})$. As mentioned earlier, this is characteristic of many reports in the literature, and is evident in Experiment 2 (see Column 1 of Figure 5).

Assuming the increasing-slope model, three-parameter exponential functions can be fit to the iso- $\Delta \mathrm{f}$ (isostimulus, or performance-based measurement) ABRM functions. As can be seen from Table 1, the estimated taus increase from $35.3 \mathrm{msec}$ for $\Delta \mathrm{f}=4 \mathrm{~Hz}(3 \times$ tau $=$ $105.9 \mathrm{msec})$ to $76.5 \mathrm{msec}$ for $12 \mathrm{~Hz}(3 \times$ tau $=$ $229.5 \mathrm{msec}$ ), and then decrease again to $42.1 \mathrm{msec}$ for $24 \mathrm{~Hz}(3 \times$ tau $=126.3 \mathrm{msec})$. Especially troublesome is that even though the underlying generating psychometric functions were defined to have tau $=36.8 \mathrm{msec}$ at $\mathrm{d}^{\prime}=1.0(3 \times \mathrm{tau}=110.4 \mathrm{msec})$, the iso- $\Delta \mathrm{f}$ functions near $\mathrm{d}^{\prime}=1.0$ (in the middle range of the proportion correct measure) of 8 to $12 \mathrm{~Hz}$ have taus of 62.5 to $76.5 \mathrm{msec}$, respectively ( $3 \times$ tau values of 187.5 to $229.5 \mathrm{msec}$ ). Presumably the nonlinearities in $\mathrm{P}(\mathrm{C})$, even in this middle range away from ceiling and floor effects, is causing this perturbation in the time constant, tau.

Taus obtained in the isoperformance (iso-d') ABRM functions under the increasing-slope model appear to be less variable across the approximately equally large range of performance levels than those in the iso- $\Delta \mathrm{f}$ functions. These taus vary from 28.0 to $49.4 \mathrm{msec}(3 \times$ tau values of 84 to $148.2 \mathrm{msec}$ ) for $\mathrm{d}^{\prime}$ values of 0.5 to 3.0. As Table 1 shows, these isoperformance duration estimates vary less from 110.4 msec $\left(d^{\prime}=1.0\right)$ than in the corresponding isostimulus case.

The constant-slope model, with underlying psychometric functions of equal slope and exponentially decreasing thresholds ( $\mathrm{d}^{\prime}=1.0$ ), yields the iso- $\Delta \mathrm{f}$ (isostimulus) and iso-d' (isoperformance) ABRM functions in Column 3 of

Table 1

Estimated Duration of ABRM for Certain Parameter Values for the Three Sets of Underlying Psychometric Functions

\begin{tabular}{cccc}
\hline & \multicolumn{2}{c}{ Set of Underlying Psychometric Functions } \\
\cline { 2 - 4 } Parameter & Experiment 2 & $\begin{array}{c}\text { Increasing- } \\
\text { Slope Model }\end{array}$ & $\begin{array}{c}\text { Constant- } \\
\text { Slope Mode] }\end{array}$ \\
\hline & Isostimulus [P(C) $\times \Delta t$ Space] \\
$\Delta f(H z)$ & & & \\
24 & 125.1 & 126.3 & 95.4 \\
20 & 152.4 & 168.0 & 113.1 \\
16 & 200.1 & 213.0 & 129.3 \\
12 & 232.5 & 229.5 & 142.5 \\
8 & 205.5 & 187.5 & 151.8 \\
4 & 139.5 & 105.9 & 156.6 \\
& Isoperformance [ $\Delta \mathrm{f} \times \Delta \mathrm{t}$ Space] \\
$\mathrm{d}^{\prime}$ & & & \\
3.0 & 155.4 & 148.2 & 110.4 \\
2.5 & 150.6 & 142.8 & 110.4 \\
2.0 & 144.0 & 135.6 & 110.4 \\
1.5 & 135.0 & 125.7 & 110.4 \\
1.0 & 121.5 & 110.4 & 110.4 \\
0.5 & 93.3 & 84.0 & 110.4 \\
\hline
\end{tabular}

Note-Duration estimates are $3 \times$ tau values from three-parameter exponential fits to the functions in the lower two rows of Figure 5.
Figure 5. When these two sets of functions are fit by a three-parameter exponential curve, the iso- $\triangle \mathrm{f} A B R M$ functions yield taus that decrease from $52.2 \mathrm{msec}$ for a $\Delta \mathrm{f}$ of $4 \mathrm{~Hz}$ to $31.8 \mathrm{msec}$ at $24 \mathrm{~Hz}$, whereas the iso-d' functions all yield a constant tau of $36.8 \mathrm{msec}$. Clearly, if the constant-slope model were assumed, measuring $P(C)$ as a function of $\Delta t$ for fixed stimulus values would yield large variations in the estimate of the duration of ABRM ( $3 \times$ tau of 95.4 to $156.6 \mathrm{msec}$ ). Presumably, because of the nonlinearities of $P(C)$, misleading values of tau would be found even if one were to avoid ceiling and floor effects by using a stimulus value of $12 \mathrm{~Hz}$. For $\Delta \mathrm{f}$ of $12 \mathrm{~Hz}$, tau $=47.5 \mathrm{msec}(3 \times$ tau $=142.5 \mathrm{msec})$, whereas in the underlying generating psychometric functions tau $=36.8 \mathrm{msec}(3 \times$ tau $=110.4 \mathrm{msec})$. In other words, with the constant-slope model, stimulus-based (isoperformance) measurement of ABRM preserves the appropriate constant value of tau, a measure of the time course of ABRM, whereas performance-based (isostimulus) measurement does not.

It appears reasonable to argue that if a quantitative measure of the time course of backward masking (tau) is more perturbed and more variable when derived from performance-based measurements (iso- $\Delta f$ ) than from stimulus-based measurements (iso-d') of ABRM, then estimates derived from "visual fits" to data must be suspect for the same reason. Because of these problems, previous interpretations of the results of ABRM experiments with $\mathrm{P}(\mathrm{C})$ as the dependent variable have, at best, provided an incomplete picture of the nature of that effect. At worst, the choice of arbitrary stimulus values for use in those experiments and nonquantitative estimates of the time course of ABRM may have severely overestimated the role of that effect in the processing of complex, spectrally varying sounds.

In summary, the ABRM effects are more clearly described by stimulus-based measurements (isoperformance) than by performance-based (isostimulus) measures. More informative than either of these is to determine the underlying generating psychometric functions.

In these experiments, the existence of ABRM effects was demonstrated using well-trained listeners and relatively low stimulus uncertainty. Those effects, when measured in stimulus terms, however, were found to be small (an increase in thresholds from about 6 to $12 \mathrm{~Hz}$ ) and of short duration (about $110 \mathrm{msec}$ ). Earlier findings of longer duration effects appear to have been strongly influenced by the particular form of data analysis employed.

\section{REFERENCES}

ACM, INC. (1976). Collected algorithms from CACM (Vol. 2, Algorithm 295). New York: Author.

Egan, J. P., Lindner, W. A., \& McFadden, D. (1966). Maskinglevel differences and the form of the psychometric function. Journal of the Acoustical Society of America, 37, 1181. (Abstract)

GREEN, D. M., SWETs, J. A. (1974). Signal detection theory and psychophysics. New York: Krieger. (Original work published 1966 by Wiley, New York.) 
Kallman, H. J., \& Massaro, D. W. (1979). Similarity effects in backward recognition masking. Journal of Experimental Psychology: $\mathrm{Hu}$ man Perception and Performance, 5, 110-128.

KALlMAN, H. J., \& MASSARO, D. W. (1983). Backward masking, the suffix effect, and preperceptual storage. Journal of Experimental Psychology: Learning, Memory, and Cognition, 9, 312-327.

Leshowitz, B., \& CUDAHY, E. (1973). Frequency discrimination in the presence of another tone. Journal of the Acoustical Society of America, 54, 882-887.

LEVITT, H. (1971). Transformed up-down methods in psychoacoustics. Journal of the Acoustical Society of America, 49, 467-477.

LOEB, M., \& HoldiNG, D. H. (1975). Backward interference by tones or noise in pitch perception as a function of practice. Perception \& Psychophysics, 18, 205-208.

MASSARO, D. W. (1970). Preperceptual auditory images. Journal of Experimental Psychology, 85, 411-417.

Massaro, D. W. (1972a). Preperceptual images, processing time, and perceptual units in auditory perception. Psychological Review, 79, 124-145.

Massaro, D. W. (1972b). Stimulus information vs. processing time in auditory pattern recognition. Perception \& Psychophysics, 12, 50-56.

MASSARo, D. W. (1973). A comparison of forward versus backward recognition masking. Journal of Experimental Psychology, 100, 434-436.

MASSARO, D. W. (1975). Backward recognition masking. Journal of the Acoustical Society of America, 58, 1059-1065.

Massaro, D. W. (1976). Auditory information processing. In W. K. Estes (Ed.), Handbook of learning and cognitive processes: Vol. 4. Attention and memory. Hillsdale, NJ: Erlbaum.

Massaro, D. W., Cohen, M. M., \& Idson, W. L. (1976). Recognition masking of auditory lateralization and pitch judgments. Journal of the Acoustical Society of America, 59, 434-441.

MASSARO, D. W., \& KaHN, B. J. (1973). Effects of central processing on auditory recognition. Journal of Experimental Psychology, 97, 51-58.

MoORE, B. C. J. (1973). Frequency difference limens for short-duration tones. Journal of the Acoustical Society of America, 54, 610-619.

SPARKs, D. W. (1976). Temporal recognition masking-or interference? Journal of the Acoustical Society of America, 60, 1347-1353.

Watson, C. S. , Jensen, J. K., Foyle, D. C., Leek, M. R., \& GoldGAR, D. E. (1982). Performance of 146 normal adult listeners on a battery of auditory discrimination tests. Journal of the Acoustical Society of America, 71, S73. (Abstract)

Watson, C. S., Kelly, W. J., \& Wroton, H. W. (1976). Factors in the discrimination of tonal patterns. II. Selective attention and learning under various levels of stimulus uncertainty. Journal of the Acoustical Society of America, 60, 1176-1186.

Yost, W. A., Berg, K., \& Thomas, G. B. (1976). Frequency recognition in temporal interference tasks: A comparison among four psychophysical procedures. Perception \& Psychophysics, 20, 353-359.

\section{NOTES}

1. The exception to these objections was reported by Massaro (1972b). That study, however, investigated the ability to identify whether the test tone was a sawtooth or a sine wave, when followed by a square wave masker, with all presented at $800 \mathrm{~Hz}$. Since the task employed constant- frequency stimuli and yielded extremely long-duration estimates of ABRM $(3 \times$ tau $=384$ to $590 \mathrm{msec}$, depending on subjects' discrimination performance), the results may not be comparable to those obtained in pitch-identification tasks.

2. Lloyd Jeffress once commented, in a similar situation, that "this convenient means of disposing of aberrant data should not go without remark." While the inability to perform at better than chance levels for any of the stimuli in Experiment 1 is rather unusual in our experience, it is not entirely without precedent. In ABRM tasks, Massaro (1972b) reports data in which subjects perform extremely poorly, and likewise, Kallman and Massaro (1979) had to discard 7 out of 18 subjects for poor performance. In our work on individual differences in auditory discrimination abilities, performance of the least sensitive $10 \%$ of 127 listeners with no identified auditory pathology was poor enough to be consistent with this result. We have found that individual differences in tasks with complex or time-varying sounds are considerably larger than those for single-tone detection or discrimination (Watson, Jensen, Foyle, Leek, \& Goldgar, 1982). It is important to remember that there do exist people who perform extremely poorly on this task, and that the present results and discussions (and most of those in the literature) pertain only to those subjects who initially show at least moderately good performance at the long values of $\Delta t$.

3. After Experiment 1 and before Experiment 2, the three listeners were tested for 500 trials $(1 \mathrm{~h})$ in a task procedurally identical to Experiment 1 but with a $\Delta \mathrm{f}$ of $24 \mathrm{~Hz}$. Since those data are tangential to the present issues, they are not reported here.

4. In light of our argument about possible stimulus uncertainty effects on ABRM, a comment should be made about stimulus uncertainty effects in a tracking procedure. It might appear that stimulus uncertainty is very high in the tracking task (with 20 possible values of $\Delta f$ and 6 of $\Delta t$ ). It can be argued, however, that the level of stimulus uncertainty in tracking tasks can be quite small. The effects of stimulus uncertainty very likely reflect difficulty in finding an optimal strategy when many different stimuli are possible on each successive trial and when each stimulus must be attended to in a different way (i.e., using different cues, different decision criteria, etc.). In an adaptive-tracking procedure with small step sizes, the subject tends to receive only a small number of different stimuli within a given time period, all of which are similar on the tracking dimension. Thus, stimulus uncertainty can be quite low over any specific trial sequence. Within such trial sequences, there probably is a single strategy that can be adopted, with optimal performance the result. With large step sizes, stimulus uncertainty can, of course, be much greater.

5. As mentioned earlier, the validity of the $\Delta f$ estimates for $\mathrm{d}^{\prime}=1.0$ is supported by their agreement with the stimulus values on which the tracking algorithm converged. The estimates of psychometric-function slope and of iso- $d^{\prime}$ functions greatly different from $d^{\prime}=1.0$ obtained in Experiment 2, however, may be less stable, since the tracking algorithm tends to present mostly stimulus values that yield approximately a $P(C)=0.707$ with proportionally fewer stimulus values tested that yield higher or lower performance. Although the fits to individual subjects' psychometric functions obtained in Experiment 2 yielded an average correlation of $\mathrm{r}=+0.74$, ranging from +0.46 to +0.96 , the variance in the slopes of the fitted functions does not allow us to adequately distinguish between the two models.

(Manuscript received January 31, 1984; revision accepted for publication October 30, 1984.) 